\title{
El derecho empresarial en un entorno de flipped classroom
}

\author{
Effect Business law in a flipped classroom \\ environment
}

\author{
Noemí Jiménez Cardona \\ Profesora-Investigadora de Derecho Mercantil \\ Miembro integrante del Grupo de Innovación Docente Dikasteia \\ Departamento de Derecho Privado \\ Universitat de Barcelona, España \\ E-mail: njimenezcardona@ub.edu
}

Resumen: El presente artículo centra su atención en la experiencia docente (innovación) que, como profesora e investigadora de la Sección de Derecho Mercantil del Departamento de Derecho Privado de la Universitat de Barcelona, he desarrollado en la disciplina de Derecho Mercantil del Grado de ADE, impartido en la Facultat de Economía y Empresa. Dicha experiencia tiene que ver con la utilización de la metodología docente activa de la clase inversa, y su complementación con la resolución de casos prácticos. Su aplicación ha resultado especialmente útil en orden a corregir la tradicional desmotivación de parte del estudiantado en el aula, su falta de asistencia a clase, así como la ausencia de implicación real de los estudiantes universitarios en un proceso de aprendizaje en el que, cada vez más, están llamados a asumir un papel protagonista bajo la guía del profesorado.

Palabras clave: Metodologías docentes activas; Aula inversa; Aprendizaje autónomo; Aprendizaje tutelado. 


\begin{abstract}
This article focuses on the high educational experience (innovation) that, as researcher of the Business Law Section of the Department of Private Law of the University of Barcelona, I have developed in the Business Law discipline of the ADE Degree, taught at the Faculty of Economics. This experience has to do with the use of the active teaching methodology of the flipped classroom, and its complementation with the resolution of practical cases. The application of this methodology has been particularly useful in order to correct the traditional demotivation on the part of the students in the classroom, their lack of attendance to class, as well as the lack of real involvement of university students in a learning process in which, each time moreover, they are called to assume a leading role under the guidance of the teaching staff.
\end{abstract}

Keywords: Active learning methodologies; Flipped classroom; Autonomous learning; Guided learning.

Sumario: 1. El papel de las metodologías docentes activas en orden a la mejora docente y el aprendizaje del alumnado universitario. 2. La potencialidad del aula inversa (flipped classroom) y su extrapolación al mundo del Derecho. 3. Experiencia personal de aula inversa en el Grado de ADE de la Universitat de Barcelona (curso académico 2019/2020). 3.1. Presentación del entorno académico y perfil del estudiantado. 3.2. Implementación de la flipped classroom como estrategia metodológica de aprendizaje. 4. Conclusiones.

\title{
1. El papel de las metodologías docentes activas en orden a la mejora docente y el aprendizaje del alumnado universitario
}

La docencia es uno de los ejes principales y señal de identidad de toda institución universitaria. Quiénes somos como universidad viene fijado, en buena medida, por qué enseñamos y cómo lo enseñamos. En pleno siglo XXI, desde la perspectiva de la política académica y docente es obligado centrar la atención en la dimensión docencia-aprendizaje (Font Ribas, 2014: 36-41). Ello es así, porque el estudiante, presente y futuro de la institución, debe ser el centro de atención de la actividad universitaria (Turull Rubinat, 2020: 3-4). Debemos conocerlo mejor y, en consecuencia, adaptar su formación, tanto en 
conocimientos como en habilidades, a las nuevas metodologías docentes activas (Vallespín Pérez, 2011: 1-10). Ello ha de repercutir, en positivo, en cuanto al cumplimiento efectivo de la exigencia de una Educación de Calidad, como así proclama el Objetivo núm. 4 de Desarrollo Sostenible de la Agenda 2030 , una de cuyas metas alude, expresamente, a la necesidad de asegurar, antes de dicha fecha, el acceso igualitario de todos los hombres y mujeres a una "formación superior de calidad, incluida la enseñanza universitaria".

Lejos de un modelo de universidad a distancia fundamentalista, pero siendo conscientes de la revolución tecnológica, así como de los nuevos perfiles de alumnado que ya nos llegan y llegarán a las aulas universitarias, resulta imprescindible tomar conciencia, sin llegar a menospreciar, per se, las clases tradicionales o clásicas (incluso las de perfil teórico o magistrales), acerca de la utilidad de las nuevas tecnologías y metodologías docentes en aras a jugar un rol destacado en la formación de las próximas generaciones de egresados (Jiménez Cardona, 2019: 6-7). De ahí la necesidad de repensar, continuamente, las clases, ya sea mediante las técnicas de la innovación docente o la revolución tecnológica (Esteve Segarra, 2016: 78-81).

Resulta imprescindible que el profesorado universitario se familiarice, cada día más, desde la óptica del protagonismo del alumnado y de las TIC, con el uso de nuevos lenguajes y metodologías docentes activas (aprendizaje basado en problemas, aprendizaje colaborativo, aprendizaje entre iguales, aprendizaje servicio, tutoría entre iguales, PBL, casos y simulaciones, aula inversa, gamificación y ludificación); el aprendizaje en entornos virtuales (LSS - Moodel, MOOC, Open Educational Resources, PLE - entornos personales de aprendizaje -, portafolios digitales, simulaciones, web 2.2/3.0/redes sociales); el aprendizaje profesional (competencias transversales, prácticas formativas, PNT procedimientos normalizados de trabajo-, TFG y TFM); la nueva visión de la evaluación (auto-evaluación, evaluación acreditativa, evaluación continua, evaluación entre iguales, rúbricas); y la toma en consideración tanto de los equipos docentes como de las llamadas learning analytics (Sánchez Vera, Solano Fernández y González Calatayud, 2016: 56-57).

\footnotetext{
${ }^{1}$ ONU (25 de noviembre de 2015). Resolución A/RES/70/1 Transformar nuestro mundo: la Agenda 2030 para el Desarrollo Sostenible. Recuperado de:

https://www.un.org/ga/search/view_doc.asp?symbol=A/RES/70/1\&Lang=S.
} 
En este contexto, la flipped classroom (aula inversa), que se enmarca en el modelo $B$ learning, es una de aquellas metodologías docentes activas que presenta un mayor potencial de aplicación en el aula universitaria (Morán, 2012: 3-5). El aula inversa, en cuanto modelo pedagógico, supone la inversión de la tradicional secuencia de las actividades educativas. Frente a la secuencia tradicional (enseñanza - estudio - evaluación) se impone otra diferente en que se parte del estudio, éste se evalúa y, finalmente, se contempla su enseñanza para con el alumnado (Medina Moya, 2013: 3). Se plantea así, de inmediato, la necesidad de analizar el nuevo rol docente en el ámbito del aprendizaje autónomo del alumno (Pérez Cabrera, 2013: 46-47). Un nuevo reparto de roles que, de hacerse bien, debe terminar por suponer, a medio plazo, una mejora sustancial tanto de la calidad docente como del aprendizaje del alumnado.

\section{La potencialidad del aula inversa (flipped classroom) y su extrapolación al mundo del Derecho}

El aula inversa se organiza, en realidad, en función del trabajo previo que corresponde al alumnado, siendo así que el profesor debe centrar su atención, mediante las llamadas hojas de actividad (Giménez Font, 2013: 17-18), en tutelar dicho trabajo autónomo mediante la preparación de lecturas o vídeos, la elaboración de cuestionarios y la resolución de dudas (Esteve Segarra, 2016: 85-87). Todo ello, además, debe repercutir en una optimización del tiempo de las clases presenciales.

Los principios fundamentales de la clase inversa tienen que ver con un entorno flexible, la cultura del aprendizaje, el contenido intencional y la presencia de un educador profesional (Sams et al., 2014: 1-2). El profesorado debe centrar su atención en la conceptualización de la propia asignatura, esto es, definir competencias, conceptualizar actividades, seleccionar las herramientas a utilizar y preparar y secuenciar los recursos orientados al aprendizaje; la planificación del programa de la asignatura y la actividad de aprendizaje; la gestión del aprendizaje de su alumnado; la dinamización del proceso de aprendizaje con un adecuado espacio tutorial personalizado y orientado a la solución de dudas; la motivación del alumnado mediante la creación de un entorno agradable de aprendizaje; y la medición 
de dicho aprendizaje mediante una evaluación continua en que la tenga cabida la retroalimentación personalizada (Sánchez Rodríguez, Ruíz Palmero, y Sánchez Vega, 2017: 343).

En el modelo de aula inversa el alumno adquiere, por tanto, un mayor protagonismo en su proceso de aprendizaje, así como también aumenta su interés e implicación en la asistencia y seguimiento de las clases que corresponden a cada disciplina (Font Ribas et al., 2012: 69). Se estimula el trabajo autónomo del alumno, guiado por su responsable docente, así como se introducen elementos dinámicos y dialécticos en el aula y se fomenta el trabajo en equipo (por ejemplo, las simulaciones de casos y la técnica del flash) (Medina Moya, 2013: 6). Unos elementos, por cierto, de notable relevancia en la formación en competencias y habilidades que son propias de los estudios jurídicos y, muy especialmente, del Derecho Mercantil (Sobrino López, 2018: 4-6), disciplina ésta de notable complejidad técnica, claramente interrelacionada con otras ramas del Derecho (como es el caso del Derecho Procesal, el Derecho Administrativo, el Derecho Penal, el Derecho Constitucional y el Derecho Internacional) y con relevancia práctica en el ámbito de la empresa y los negocios.

El aula inversa constituye una herramienta docente, favorecida en buena medida por la nueva cultura virtual, híbrida o mixta, que viene amparada por la utilización de los campus virtuales (exponencialmente utilizados con motivo de la actual crisis pandémica derivada de la Covid-19, cuyos incipientes problemas son puestos de relieve por Andrés Aucejo, 2020: 1-8) y en la que el profesorado transfiere fuera del aula parte de la información que debe conocer el alumnado, pero sin renunciar a su condición de guía del aprendizaje (Bergmann y Sams, 2012: 13-14). En una universidad presencial, como lo es y ha de seguir siendo la Universitat de Barcelona, parece conveniente recordar, de una parte, que la universidad es un lugar afectivo que va mucho más allá de recibir clases; y, de otra, que el contacto personal y directo con el profesorado jamás debe sustituirse, en su integridad, por un teclado o una pantalla de ordenador.

La implementación del aula inversa puede afrontarse, en función de las particularidades que son propias de cada disciplina jurídica (principio de flexibilidad), con diferentes 
metodologías que van desde el just in time teaching ${ }^{2}$, hasta el team based learning ${ }^{3}$, pasando por el flipped learning + peer instruction ${ }^{4}$, la flipped mastery learning ${ }^{5}$, la flipped

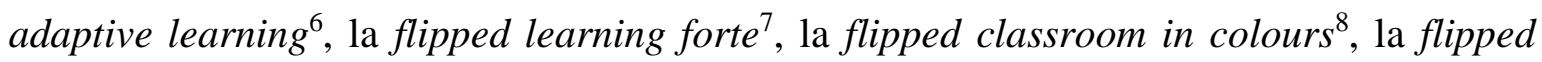
learning acompañada de gamificación ${ }^{9}$, el aprendizaje colaborativo ${ }^{10}$, el aprendizaje basado en problemas ${ }^{11}$ o el conocido como aprendizaje servicio ${ }^{12}$.

\footnotetext{
${ }^{2}$ En esta estrategia de aprendizaje los alumnos realizan una tarea antes de la clase y el profesor, tras analizar sus respuestas, procede a la adaptación, en el momento justo y adecuado, de los materiales y actividades educativas para así facilitar que puedan obtener la respuesta adecuada a sus necesidades formativas.

${ }^{3}$ En este aprendizaje basado en equipos, el proceso de enseñanza-aprendizaje se inicia con una actividad de estudio previa, guiada por el profesor, que cada alumno debe desarrollar de forma individual. Más tarde, ya en el aula, se persigue asentar, en primer lugar, los conocimientos por parte del alumnado. A continuación, se realiza una "puesta en común" y el profesor suele terminar su actividad con una mini-clase valorativa de las respuestas suministradas por el alumnado, así como con el planteamiento de algunas actividades grupales sobre problemas significativos que guarden relación con temas de actualidad de su disciplina.

${ }^{4}$ La peer instruction consiste en responder una cuestión planteada por el docente mediante el debate, la discusión y la explicación de los diferentes puntos de vista del alumnado.

${ }^{5}$ En esta estrategia formativa los estudiantes antes de poder pasar al nivel siguiente de formación, deben demostrar que han alcanzado un nivel de perfeccionamiento sobre los conocimientos previos, siendo así que los contenidos de la disciplina se organizan, por el profesor, mediante la fijación de objetivos específicos.

${ }^{6}$ En este aprendizaje, de naturaleza adaptativa, se interacciona de forma directa entre profesor y alumno, haciendo así posible la adaptación de los diferentes recursos, así como de las actividades, a las que constituyan, en cada caso concreto, las necesidades específicas de formación.

${ }^{7}$ Evolución del just in time teaching en la que los alumnos reciben, en primer lugar, la información y la asimilan fuera del aula; para, acto seguido, remitir sus dudas al profesor de forma directa. Recibidas dichas dudas, el docente las contesta no solo a quien las ha formulado, sino a todos los integrantes del grupo o clase.

8 Esta metodología se caracteriza porque los alumnos revisan los materiales en casa y contestan al cuestionario planteado por el profesor, aprovechando la ocasión para enviarle sus dudas marcadas por colores (por ejemplo, en negro las que precisen la explicación del profesor; en verde aquéllas que puedan ser contestadas por sus propios compañeros de clase, en rojo las que denoten una clara falta de comprensión de lo estudiado de forma autónoma, o en naranja las que puedan servir para abrir un debate enriquecedor sobre la temática objeto de estudio).

${ }^{9}$ Se acompaña el aula inversa de elementos de gamificación, esto es, de diseños de juegos que pueden incrementar la motivación del alumnado respecto a una determinada disciplina [vid. JIMÉNEZ CARDONA, N. (2019). Reflexiones acerca de una experiencia de "gamificación" en Derecho mercantil dentro del grado de ADE de la Universitat de Barcelona. Revista Educación y Derecho - Education and Law Review, (21), 113].

${ }^{10}$ Se aprovecha el aula inversa para fomentar en el aula el trabajo en equipo.

${ }^{11}$ Metodología de aprendizaje basada en el planteamiento de un problema real al estudiante, siendo éste quien deberá asumir la responsabilidad de investigar y auto-suministrarse los conocimientos necesarios para alcanzar su solución. Sobre la implementación de esta metodología en el ámbito jurídico nos remitimos a FONT RIBAS et al. (2009). Las dificultades del aprendizaje autónomo en un contexto de ABP. Revista Educación y Derecho - Education and Law Review, (1), 1-26.

${ }^{12}$ Modalidad de aprendizaje en el que se combina la formación en contenidos, competencias y valores, con aquella otra basada en el aprendizaje experiencial y el desarrollo de la responsabilidad cívica y social de los estudiantes, los cuales se implican en la realización de tareas en favor de la propia sociedad [vid. ESCOFET ROIG, A. (Ed.). (2017). Aprendizaje-servicio (ApS): claves para su desarrollo en la universidad. Barcelona: Octaedro].
} 
En cualquier caso, el profesorado implicado en la flipped classroom debe no solo dedicar parte de su tiempo a pensar y diseñar la planificación de su estrategia educativa en el aula inversa, sino también a determinar cuál sea el grado de información del alumnado en el caso concreto (Medina y Jarauta, 2013: 23-28) y, en consecuencia, implementar diferentes acciones que permitan su aprendizaje por la vía de la retroalimentación (Balslev, Vanhulle y Tominska, 2011: 23-29). Un proceso en el que será necesario, en todo momento, contar con la implicación activa de los estudiantes en orden a construirse su propio estudio previo sobre la materia (Medina Moya, 2013: 6), superando así la comodidad de la copia de apuntes o el simple manejo y reproducción de contenidos de un manual.

El éxito del aula inversa requiere de una doble concienciación y motivación: la del profesorado y la del propio alumnado (Espel Masferrer, 2013: 7-15). El profesor debe ser consciente de la importancia que cabe atribuir al trabajo previo y dirigido a la preparación y diseño de los materiales docentes (elección de lecturas, elaboración de cuestionarios y preparación de clases dinámicas).

Este esfuerzo de planificación también debe acompañarse de una meditada reflexión acerca del modelo de evaluación, pues parece evidente que la clase invertida no se adecúa bien a un sistema de evaluación de tipo meramente memorístico. En este sentido conviene apostar, sin duda, por la generalización de un modelo de evaluación continua en el que se ponderen, en su justa proporción, el trabajo de estudio previo del alumno, la repuesta de los cuestionarios y su participación activa en unas clases en las que la transmisión del conocimiento se centra, no ya tanto en el profesor, como en el propio alumnado (Esteve Segarra, 2016: 89-93). Todo ello, como es lógico, no es incompatible, según la disciplina, con la previsión de un examen final teórico-práctico en el que se permita evaluar las diferentes competencias jurídicas adquiridas por los propios estudiantes (vgr. identificación del problema o cuestión base, manejo de las fuentes jurídicas, extracción de la interpretación normativa, y, por último, resolución y fundamentación del problema).

Si tanto el profesor como el alumno se implican, de verdad, en el aula inversa, aumenta su motivación, su constancia y, por extensión, la solidez de sus conocimientos (quizás sea bueno analizar también, llegados a este punto, el actual volumen de contenidos que son 
propios de un determinado plan de estudios y apostar, en paralelo, no tanto por su cantidad, como por su calidad).

Particularmente, como luego analizaré con detalle en el posterior apartado relativo a la experiencia docente, el seguimiento de una metodología activa como la flipped classroom coadyuva, sin duda, a incrementar esa potencialidad. Una potencialidad que, en todo caso, al menos hasta que la cultura del profesorado y, en especial del alumnado, mute, debiera compatibilizarse con algunas clases magistrales, de perfil tradicional. De no hacerlo así, corremos el riesgo de sobrecargar de trabajo a un alumnado no preparado para ello y, de rebote, provocar su desánimo y rechazo a una disciplina en concreto, máxime si el resto de asignaturas de la titulación de referencia siguen respondiendo al perfil de una docencia fundada en un aprendizaje más clásico (Sánchez Vera, Solano Fernández y González Calatayud, 2016: 58-60).

Si sabemos combinar, en la realidad actual, la clase tradicional con el aula inversa es casi seguro que, a medio plazo, los alumnos no solo estarán deseando ser la pieza clave de su propio aprendizaje, sino que también reclamarán profesores que sepan planificar su estudio previo a la discusión, así como también el debate de los diferentes temas de relevancia en el aula universitaria (Sánchez Rodríguez, Ruíz Palmero, y Sánchez Vega, 2017: 339-340). Unos alumnos llamados, además, a desarrollar un trabajo colaborativo en el aula que, por extensión, fomente su desafío intelectual e interés por una determinada asignatura (Bennett et al., 2011: 9-12).

\section{Experiencia personal de aula inversa en el Grado de ADE de la Universitat de} Barcelona (curso académico 2019/2020)

\section{1.- Presentación del entorno académico y perfil del estudiantado}

Centrando la reflexión en el entorno de las ciencias económicas y jurídicas, cabe señalar que la técnica del aula inversa, aun de forma inconsciente, siempre ha estado presente en 
las aulas universitarias, bien a través de la preparación previa de casos prácticos o, en su caso, mediante el debate grupal sobre temas de actualidad que, previamente, han sido preparados por los propios alumnos a través de materiales específicos (vgr. artículos doctrinales, resoluciones judiciales, notas de prensa, borradores de proyectos normativos). Con todo, es obligado señalar, de una parte, que la proliferación de grupos especialmente numerosos (de 70 a 100 alumnos) en este ámbito de conocimiento no siempre hace sencilla su implementación práctica; y, de otra, que los nuevos recursos tecnológicos, así como las posibilidades que nos abre la utilización del campus virtual (Turull Rubinat, 2020: 3-4) están llamados a facilitar el tránsito de no pocos docentes, hasta ahora reacios, hacia la utilización, sino generalizada, cuando menos puntual, de la metodología del aula inversa (Sánchez Vera, Solano Fernández y González Calatayud, 2016: 66).

Ante esta realidad y en lo que constituye la asignatura de Derecho Mercantil del Grado de ADE, detallaré a continuación cuál ha sido la experiencia docente sobre el uso y dinámica del aula inversa; debiendo hacer constar, desde ya, que ha resultado especialmente útil para combatir la clásica desmotivación de parte del estudiantado, así como para hacer más amena la complejidad propia de una rama jurídica que, además, tiene claras connotaciones económicas y empresariales (Vázquez Albert et al., 2011: 49-51).

Antes de atender a la estructuración de la docencia de esta asignatura mediante la metodología de la flipped classroom, muy en particular en lo que se refiere al diseño de los contenidos y materiales destinados a los alumnos en sus horas de aprendizaje autónomo, es necesario tomar en consideración su contexto académico. Si en algo destaca la asignatura de Introducción al Derecho Mercantil (IDE) es por tratarse de una materia compleja y un tanto conflictiva dentro del íter curricular de los planes de estudios del grado de Administración y Dirección de Empresas. Con frecuencia, la impartición de esta asignatura viene acompañada de datos no demasiado alentadores, como así sucede con las tasas de éxito, seguimiento y rendimiento académico, todas ellas situadas por debajo del promedio del resto de materias que integran la titulación ${ }^{13}$.

${ }^{13}$ De conformidad con los indicadores proporcionados por la Universitat de Barcelona VSMA 2017 relativos al Grado de Administración y Dirección de Empresas, la tasa media de abandono de la asignatura de 
Gran parte de estos inconvenientes bien pudiera obedecer, por un lado, al extenso y ambicioso alcance de contenidos que prevé el propio plan docente de la asignatura, y por otro, a las especificidades y necesidades de formación que requiere el perfil de alumno que cursa este tipo de formación universitaria. En la mayoría de casos, los estudiantes de IDE presentan, de por sí, un perfil que podría considerarse crítico. Al tratarse de alumnos de recién ingreso, a la mayoría de ellos les resulta especialmente difícil acostumbrarse a trabajar, en un primer momento, de forma autónoma, máxime cuando se trata de una asignatura jurídica que se imparte en un grado, el de ADE, en el que predominan los conocimientos propios de las matemáticas, la economía y la estadística.

También tiene que tenerse en cuenta que en el grado de Administración y Dirección de Empresas coexiste una tipología muy variada de estudiantes, procedentes tanto de las PAU y Bachillerato como de la formación profesional, por lo que la edad, experiencia e intereses académicos/laborales suelen ser muy dispares. De otra parte, la motivación que tienen los estudiantes, en general, por los contenidos de la asignatura es significativamente baja, pues parten del prejuicio, equivocado, de que todo lo jurídico es ajeno, por definición, al ámbito profesional de la empresa y los negocios. Además, en los casos de estudiantes que han cursado algún grado de formación profesional y han tenido asignaturas jurídicas la percepción suele empeorar debido a malas experiencias anteriores relacionadas con la dificultad o extensión de la materia.

\section{2.- Implementación de la flipped classroom como estrategia metodológica de aprendizaje}

Ante esta realidad, se ha optado, en cuanto estrategia metodológica de aprendizaje, por combinar el uso y dinámica del aula invertida o flipped classroom con la incorporación, dentro de las sesiones de trabajo presencial en clase, del aprendizaje basado en problemas

Introducción al Derecho de la Empresa es ligeramente elevada de tenerse en cuenta la tasa de abandono global de la titulación (29.45\%). Lo mismo sucede en caso de compararse la tasa media de eficiencia de la asignatura $(72.78 \%$ ) con la tasa de eficiencia de la titulación $(86.60 \%)$. A su vez, dentro del $91.43 \%$ de alumnos que finalizaron en 2017 la asignatura de IDE, el 58.14\% lo hicieron con aprobado, el $28.63 \%$ con notable, el $10.71 \%$ suspendió y sólo 1' $85 \%$ obtuvo la calificación de excelente. 
para aquellos alumnos que hayan decidido permanecer en el régimen de evaluación continuada. Bien puede anticiparse que la sustitución de las clases magistrales por la aplicación simultánea y continuada de ambas fórmulas ha contribuido a generar una dinámica capaz de invitar al alumno a mantener un proceso de aprendizaje activo y continuado en el tiempo. Ello ha resultado muy útil para combatir la clásica desmotivación de parte del estudiantado, así como la dificultad y complejidad propia de una materia jurídica con notables implicaciones en el terreno de la economía y el mundo de la empresa.

En lo que concierne a la implementación de la flipped classroom en esta asignatura, debe tenerse en cuenta que, con el objetivo de concebir un ambiente agradable y facilitador del autoconocimiento, el diseño de dicha metodología activa ha partido de la combinación de diferentes recursos de naturaleza online y offline. Con carácter previo a la sesión presencial en el aula, los estudiantes debían preparar los contenidos de cada unidad con los diferentes materiales que tenían a su disposición en el Campus Virtual. Dicha tarea ha comprendido la reproducción de un vídeo explicativo sobre la unidad didáctica correspondiente (todos ellos han sido elaborados por el propio profesor mediante el programa Screen-o-matic y en su visualización se ha acompañado de materiales de soporte y seguimiento). Dicho proceso de aprendizaje activo también ha requerido que el alumno se imprimiese un cuaderno de seguimiento, de carácter evaluable, en el que debía autocompletar sus recuadros y casillas con los contenidos más relevantes de cada tema. Los cuadernos de seguimiento no han tenido un contenido reglado, sino que cada alumno ha podido elaborarlos de la forma que haya entendido más cómoda para su estudio (ya fuera ayudándose de gráficos, esquemas o de tiras de visual thinking). En paralelo, se les ha recomendado que complementara sus notas con lo dispuesto en las fuentes normativas y jurisprudenciales que fueren relevantes en cada caso concreto.

Con el objetivo de evitar que el alumnado asumiese un rol pasivo y limitado a la mera visualización del contenido de la grabación, los materiales offline y online no se han estructurado de forma secuencial, sino que los vídeos han sido diseñados para compaginar la explicación de los conceptos básicos y el trabajo autónomo del estudiante en su propio cuaderno de seguimiento. Esta cuestión organizativa ha sido especialmente positiva en cuanto ha incrementado, relativamente, la sensación de acompañamiento en las horas 
asignadas de trabajo autónomo, así como también ha servido para que los estudiantes empezaran a tomar contacto y manejar, por su propia cuenta y riesgo, el material normativo con el que posteriormente deberían resolver los supuestos problemáticos planteados en clase.

Tras la asimilación inicial de los contenidos de manera autónoma y previa a cada sesión, los estudiantes han debido realizar, con un mínimo de tres horas de antelación a que tuviera lugar la sesión presencial, un cuestionario virtual de carácter evaluable y disponible en el Campus Virtual. Estas pruebas se han compuesto de diez ítems, los cuales, en función de los contenidos a tratar en cada unidad, podían presentarse en forma de pregunta con respuesta múltiple, enunciados con verdadero o falso, o también podían consistir en relacionar dos bloques de conceptos con definiciones o con ejemplos.

Solo tras efectuar las sesiones de trabajo autónomo e individual por parte de cada uno de los alumnos, ha tenido lugar el trabajo presencial mediante la presentación y resolución de un escenario-problema. La resolución de estos supuestos prácticos se ha llevado a cabo en dos sesiones presenciales semanales y mediante el trabajo en equipos estables y formados por tres o cuatro miembros.

En la primera sesión presencial se ha hecho entrega de un dossier en el que se planteaba un escenario-problema y en el que además se combinaba, según el contenido de cada unidad, la reproducción de recursos controvertidos que giraran en torno a dicha problemática (vgr. canciones, anuncios publicitarios, fragmentos de obras literarias), o bien la entrega de documentación jurídica (vgr. contratos, resoluciones jurisdiccionales, dictámenes periciales).

La distribución de estas sesiones presenciales, más enfocadas a la formación de contenidos a cargo de los propios estudiantes, han venido precedidas de un planteamiento inicial del caso por parte del profesor, así como de la elaboración de un esquema sobre los conceptos clave a tener en cuenta en orden a su discusión (veinte minutos). Tras este breve punto de partida, con el que se pretende dar respuesta a dudas referidas al marco y contexto general del caso, ha correspondido a los estudiantes, divididos por equipos de trabajo, poner en 
común y debatir entre ellos los contenidos teóricos de la unidad correspondiente, para después alcanzar una posición unánime y fundamentada capaz de resolver la situación planteada en el propio dossier.

Para cumplir con este objetivo didáctico, al finalizar la primera sesión presencial cada equipo de trabajo debía hacer entrega de un borrador, en el que además de contener una propuesta inicial de resolución, se identificaran con detalle los diferentes escenarios bajo los que podría enfocarse el problema y se señalara todo el abanico de preceptos legales que debieran tenerse en cuenta en aras a alcanzar una solución fundamentada y acorde a la normativa.

En paralelo a la finalización de la primera sesión presencial, la profesora o profesor debía poner a disposición, dentro del Campus Virtual, materiales de estudio complementarios sobre el tema en cuestión. De este modo, los estudiantes tenían la posibilidad de incrementar los conocimientos sobre los conceptos básicos ya aprendidos e incorporar, con su correspondiente anotación en el cuaderno de seguimiento, nuevos matices para la posterior resolución del caso.

En la segunda sesión presencial, los equipos de trabajo debían poner en común y debatir la nueva información que, en su caso, hubieran analizado, y preparar las conclusiones definitivas que hubieren alcanzado sobre el problema planteado inicialmente en clase. Finalmente, la segunda sesión presencial concluía con la entrega de la propuesta definitiva de resolución por cada equipo de trabajo y con la celebración de un pequeño debate guiado sobre los aspectos que hubieran resultado más controvertidos o confusos en el momento de su resolución.

\section{Conclusiones}

La flipped classroom, como metodología de naturaleza activa en la que el alumno está llamado a asumir un compromiso más participativo en su proceso de aprendizaje, no solo es útil para consolidar los conocimientos exigidos por la propia materia, sino también para fomentar la adquisición de competencias y habilidades necesarias y enfocadas al ejercicio 
de su profesión. En este contexto, el empleo del aula invertida ha demostrado ser una herramienta clave para contrarrestar alguno de los tradicionales puntos críticos que todo docente debe enfrentar a diario en el aula universitaria (desmotivación, falta de asistencia a clase o ausencia de implicación del alumnado).

En lo que concierne a mi experiencia docente, debo significar que el diseño e implementación de la flipped classroom me ha permitido combatir el tan temido problema de la extensión de contenidos del plan docente. La combinación conjunta de la flipped classroom con el aprendizaje basado en problemas, desarrollado en las sesiones presenciales, me ha permitido reducir algunos de los principales inconvenientes que comúnmente acompañaban a la impartición de esta asignatura, así como racionalizar la carga de trabajo del alumnado.

Como bien puede inferirse de las diferentes fuentes de datos recopiladas durante el curso académico (vgr. cuestionarios de incidencias críticas, cuadernos de seguimiento, listas de control de asistencia, número de entregas y continuidad de las actividades de evaluación continua), así como el feedback facilitado por los propios alumnos durante la puesta en común y resolución conjunta de las prácticas, es posible apreciar cómo la combinación de ambas metodologías ha coadyuvado, de una parte, a que los estudiantes hayan mantenido un proceso de aprendizaje continuado y sostenido en el tiempo; y, de otra, a incrementar el rendimiento académico del estudiantado no solo en las calificaciones finales sino también en todos los elementos valorativos de la evaluación continua (resolución de los escenarioproblemas, cuadernos de seguimiento y cuestionarios virtuales).

La valoración positiva que puede extraerse de la implementación de la flipped classroom, combinada con el planteamiento de escenario-problemas, viene respaldada por una pluralidad de datos concurrentes.

El número de estudiantes que ha decidido permanecer en el régimen de evaluación continuada es bastante superior al del año anterior (en el curso académico pasado, de un total de 81 alumnos únicamente 56 de ellos acabaron manteniéndose en dicha modalidad de evaluación, mientras que en el curso de referencia, del total de 84 alumnos, fueron 73 
los que permanecieron en ese mismo régimen de evaluación); así como las nueve solicitudes de cambio de modalidad de evaluación de este curso, lejos de fundarse en la sobrecarga de trabajo, lo han hecho en motivos de carácter laboral.

De otra parte, también puede constatarse un incremento significativo del rendimiento en las actividades de evaluación continua (la nota media de todas las actividades del curso anterior se situaba en un 6.20 mientras que en el curso actual ha alcanzado el 7.38), así como en la mejora de las calificaciones de la actividad final de síntesis (de un 5.15 del curso anterior hasta alcanzar un 6.40 en el curso docente actual).

La combinación del aula invertida con la resolución de escenario-problemas a través de equipos de trabajo, ha permitido que los alumnos tomen conciencia y se involucren más en su propio proceso de aprendizaje y que, en definitiva, se vean abocados a diseñar y mantener un plan de estudio continuado y periódico de los contenidos que deben trabajarse a lo largo de las diferentes prácticas. Además, la combinación de ambas fórmulas me ha permitido crear un entorno de trabajo adecuado en el que se les permite desarrollar $-\mathrm{y}$ evaluar- algunas de las competencias transversales referidas a habilidades tales como el fomento de la capacidad de razonamiento y pensamiento crítico, el desarrollo del aprendizaje autónomo y la auto-organización, o la capacidad de resolución de problemas y la toma de decisiones.

\section{Referencias bibliográficas}

ANDRÉS AUCEJO, E. (2020). Educación Superior y COVID-19: A propósito de Derechos y Garantías Fundamentales. Revista de Educación y Derecho - Education and Law Review (Covid-19: Docencia online y protección de derechos y garantías fundamentales), (22), 1-8.

BALSLEV, K., VANHULlE, S. y TOMINSKA, E. (2011). A discursive Approach to Recognition in the Practicum. McGill Journal of Education, 46 (1), 23-39. 
BENNETT, B. et al. (2012). The flipped class: What does a good one look like?. The Daily Riff: Learning, Innovation, \& Tech, (3), 1-13.

BERGMANN, A. y SAMS, A. (2012). Flip Your Classroom: reach every student in every class every day. Washington: ASCD.

ESCOFET ROIG, A. (Ed.). (2017). Aprendizaje-servicio (ApS): claves para su desarrollo en la universidad. Barcelona: Octaedro.

ESPEL MASFERRER, E. (2013). L'aula inversa: augmentant l'interès dels alumnes pel tema d'estudi. Universitat de Barcelona: Metodologies docents actives $i$ TIC per a la millora docent i de l'aprenentatge Recuperado de: www.ub.edu/portal/web/metodologies

ESTEVE SEGARRA, A (2016). Flipped Teaching o la clase invertida en la enseñanza del Derecho. Actualidad Jurídica Iberoamericana, (4 bis extraordinario), 78-81.

FONT RIBAS, A. et al. (2009). Las dificultades del aprendizaje autónomo en un contexto de ABP. Revista Educación y Derecho - Education and Law Review, (1), 1-26.

FONT RIBAS, A. et al. (2012). Las competencias genéricas en el Grado de Derecho y su evaluación. Revista de Educación y Derecho - Education and Law Review (Ejemplar dedicado a: Estrategias de formación inicial de Jueces y Magistrados), (6), 1-23.

FONT RIBAS, A. (2014). La enseñanza del Derecho tras Bolonia. REDU: Revista de Docencia Universitaria, 12 (3 extra), 36-41.

GIMÉNEZ FONT, X. (2013). L'aula inversa: una forma activa d'aprenentatge tutelat. Universitat de Barcelona: Metodologies actives $i$ TIC per a la millora docente $i$ de l'aprenentatge. Recuperado de: www.ub.edu/portal/metodologies. 
JIMÉNEZ CARDONA, N. (2019). Reflexiones acerca de una experiencia de “gamificación" en Derecho Mercantil dentro del grado de ADE de la Universitat de Barcelona. Revista Educación y Derecho - Education and Law Review, (21), 1-13.

MEDINA MOYA, J. (2013). L'aula inversa. Universitat de Barcelona: Metodologies actives $i$ TIC per a la millora docent $i$ de l'aprenentatge. Recuperado de: www.ub.edu/portal/web/metodologies

MEDINA MOYA, J. y JARAUTA, B. (2013). Enseñanza y aprendizaje en la educación superior, Madrid: Síntesis.

MORÁN, L. (2012). Blended-learning. Desafío y oportunidad para la educación actual. Edutec: Revista Electrónica de Tecnología Educativa, (39), 1-19.

PÉREZ CABRERA, L. (2013). El rol del docente en el aprendizaje autónomo: la perspectiva del estudiante y la relación con su rendimiento académico. Revista Diálogos, (11), 45-62.

SAMS, A. et al. (2014). The four pillars of F-L-I-P. Flipped Learning Network. Recuperado de: https://flippedlearning.org/wpcontent/uploads/2016/07/FLIP handout_FNL_Web.pdf

SÁNCHEZ RODRÍGUEZ, J., RUÍZ PALMERO, J. y SÁNCHEZ VEGA, E. (2017). Flipped classroom. Claves para su puesta en práctica. EDMETIC (Ejemplar dedicado a: Educación mediática y competencia digital), 6 (2), 336-358.

SÁNCHEZ VERA, M., SOLANO FERNÁNDEZ, I. y GONZÁLEZ CALATAYUD, V. (2016). FLIPPED-TIC: Una experiencia de Flipped Classroom con alumnos de Magisterio. RELATEC: Revista Latinoamericana de Tecnología Educativa, 15 (3), 55-67. 
SOBRINO LÓPEZ, D. (2018). Flipped classroom en ciencias sociales. Iber: Didáctica de las ciencias sociales, geografía e historia, (90), 4-6.

TURULL RUBINAT, M. (2020). Docencia en tiempos de Covid19. Revista de Educación y Derecho - Education and Law Review (Covid-19: Docencia online y protección de derechos y garantías fundamentales), (22), 1-5.

VALLESPÍN PÉREZ, D. (2011). El método del caso como instrumento de aprendizaje en el Derecho Procesal. Revista Educación y Derecho - Education and Law Review, (3), 1-10.

VÁZQUEZ ALBERT, D. et al. (2011). La enseñanza reflexiva del Derecho Mercantil: el diálogo reflexivo y la interrogación didáctica, en M. Turull (coord.). Experiencias de mejora e innovación docente en el ámbito del Derecho. Barcelona: Octaedro, 49-59. 